\title{
Potential Energy Surface-Based Automatic Deduction of Conformational Transition Networks and Its Application on Quantum Mechanical Landscapes of D-Glucose Conformers
}

\author{
Hiroko Satoh,, a,b,c,d Tomohiro Oda, ${ }^{\mathrm{e}}$ Kumiyo Nakakoji, ${ }^{\mathrm{f}}$ Takeaki Uno, ${ }^{\mathrm{c}}$ Hiroaki Tanaka, ${ }^{\mathrm{g}}$ Satoru Iwata,g, \\ Koichi Ohno ${ }^{\text {d,h }}$ \\ a. Research Organization of Information and Systems (ROIS), Tokyo 105-0001, Japan \\ b. Department of Chemistry, University of Zurich, 8057 Zurich, Switzerland \\ c. National Institute of Informatics (NII), Tokyo 101-8430, Japan \\ d. Institute for Quantum Chemical Exploration (IQCE), Tokyo 108-0022, Japan \\ e. Software Research Associates Inc., Tokyo 171-8513, Japan \\ f. Center for the Promotion of Interdisciplinary Education and Research, Kyoto University, Kyoto 606-8501, Japan \\ g. Department of Mathematical Informatics, University of Tokyo, Tokyo 113-8654, Japan \\ h. Department of Chemistry, Graduate School of Science, Tohoku University, Sendai 980-8578, Japan
}

\section{S1. Input files of GRRM calculations}

The conformational search calculations were carried out using the GRRM11 program package. All QM calculations were performed using the restricted Hatree-Fock (RHF) method at the 6-31G level with the Gaussian09 program package. All input files of GRRM used in the study are listed in S1-1 and S1-2. The GRRM program makes an input file of Gaussian09 and runs the process.

S1.1. GRRM input file for a-D-glucose (1)

\# GRRM/RHF/6-31G

$\begin{array}{ll}0 & 1\end{array}$

$\begin{array}{lr}\mathrm{C} & 1.187878491869 \\ \mathrm{C} & 1.529358662431 \\ \mathrm{C} & 0.484063191828 \\ \mathrm{C} & -1.210911554151 \\ \mathrm{C} & -0.219463163041 \\ \mathrm{H} & 1.570381253164 \\ \mathrm{H} & 1.253341440325 \\ \mathrm{H} & -1.198021409338 \\ \mathrm{H} & -0.278858908281 \\ \mathrm{O} & -0.796398047732 \\ \mathrm{O} & 2.771904209975 \\ \mathrm{H} & 3.486231799390 \\ \mathrm{O} & 2.163101330189 \\ \mathrm{H} & 2.043763664773 \\ \mathrm{C} & -2.627841338257 \\ \mathrm{H} & -3.047540573525 \\ \mathrm{H} & -2.628323172292 \\ \mathrm{O} & -3.471629320799 \\ \mathrm{H} & -3.093492531431 \\ \mathrm{H} & 0.606924570178 \\ \mathrm{O} & 0.534884896613 \\ \mathrm{H} & 1.424674465159 \\ \mathrm{O} & -0.434187414016 \\ \mathrm{H} & -1.138744543031 \\ \mathrm{Options} & \end{array}$

0.777001674843

$-0.512542344767$

$-1.581592564046$

0.127555977514

1.235159637678

$-0.324872433549$

0.622749060715

$-0.074735838793$

1.478350789278

$-1.061299893662$

$-1.035503337831$

$-0.412557155150$

1.733601160077

2. 576126399710

0.379680161817

1. 239784258240

0.554597704137

$-0.715742796370$

$-1.542972082527$

$-2.435385339129$

$-1.962554241633$

$-2.151638055596$

2. 410045347562

2. 972571911481
0.162868526731

$-0.557144231747$

$-0.271336071929$

0.170384385754

$-0.169159890230$

$-1.622156813254$

1. 231809412447

1. 230740384395

$-1.224985803674$

$-0.547517662716$

$-0.093814331737$

$-0.186747419214$

$-0.250729155457$

0.176642174286

$-0.297702385132$

0.200932520002

$-1.368176131645$

0.031364325995

$-0.253283488852$

$-0.915673879015$

1. 088016059840

1.374036605994

0.623713922592

0.323704946566 


\section{$\mathrm{LADD}=5$}

NLowest $=40$

Gaumem $=1000$

GauProc $=2$

Bond Condition

$12<1.82$

$15<1.82$

$17<1.30$

$113<1.71$

$23<1.83$

$26<1.30$

$211<1.71$

$310<1.69$

$320<1.29$

$321<1.70$

$45<1.83$

$48<1.30$

$410<1.74$

$415<1.82$

$59<1.30$

$523<1.72$

$1112<1.14$

$1314<1.14$

$1516<1.29$

$1517<1.30$

$1518<1.71$

$1617<2.12$

$1819<1.14$

$2122<1.14$

$2324<1.14$

End

S1.2. GRRM input file for b-D-glucose (2)

\# GRRM/RHF/6-31G

$\begin{array}{ll}0 & 1\end{array}$

C $\quad 1.119641275926$

C $\quad 1.584699130560$

C $\quad 0.585416001716$

C $\quad-1.232924102061$

C $\quad-0.288440797977$

$\mathrm{H} \quad 1.689097495155$

$\mathrm{H} \quad 1.138509544289$

$\mathrm{H} \quad-1.249572548359$

$\mathrm{H} \quad-0.311207987353$

$\mathrm{H} \quad 0.604544916311$

o $\quad-0.735631614854$

O $\quad 0.884859390622$

$\mathrm{H} \quad 0.326681428198$

O 2.812922555005

$\mathrm{H} \quad 3.513229575698$

O $\quad 2.059394100796$

$\mathrm{H} \quad 1.889404108730$

O $\quad-0.610658294663$

$\mathrm{H} \quad-1.272502360148$

C $\quad-2.647832166280$

$\mathrm{H} \quad-3.125876840092$

$\mathrm{H} \quad-2.626108585583$

O $\quad-3.440531763878$

$\mathrm{H} \quad-3.058058461756$

Options

$\mathrm{LADD}=5$
0.959202902512 $-0.432310502624$ $-1.482632030343$

0.187890943577

1.251946874438

$-0.489334998225$

1.046055085802

0.231880926468

1.251689880956

$-1.595058182548$

$-1.093263820573$

$-2.677116863722$

$-3.401986505608$

$-0.722216010068$

$-0.144294290029$

1.866869150244

2.768191986598

2. 552745563000

3. 023316045179

0.265711702469

1. 177741009613

0.235427471488

$-0.796529505519$

$-1.643885833084$
0.204935822817

$-0.189837732814$

0.245654600538

0.271471521012

$-0.271524279275$

$-1.264964959008$

1. 285978662415

1. 355214467729

$-1.355538665678$

1. 323270101510

$-0.158740331885$

$-0.402885963701$

$-0.142734174044$

0.461197597693

0.175426598409

$-0.365984618824$

$-0.111028293038$

0.238601583914

$-0.254491640414$

$-0.260648860791$

0.059905890397

$-1.344360168794$

0.255331266378

0.048763575454 
$12<1.82$

$15<1.82$

$17<1.30$

$116<1.71$

$23<1.82$

$26<1.30$

$214<1.70$

$310<1.30$

$311<1.72$

$312<1.67$

$45<1.83$

$48<1.30$

$411<1.73$

$420<1.82$

$59<1.30$

$518<1.72$

$1213<1.14$

$1415<1.14$

$1617<1.14$

$1819<1.14$

$2021<1.29$

$2022<1.30$

$2023<1.71$

$2122<2.12$

$2324<1.14$

End

\section{S2. GRRM calculation with larger LADD}

The GRRM calculation with larger LADD was carried out for $\alpha$-D-glucose (1) with LADD=10. Other contents of the input file is the same as that with LADD $=5$ shown in S1-1. Conformational types plotted against the potential energy relative to $\mathbf{1} \mathbf{a}_{\min }$ are shown in Figure S2-1.

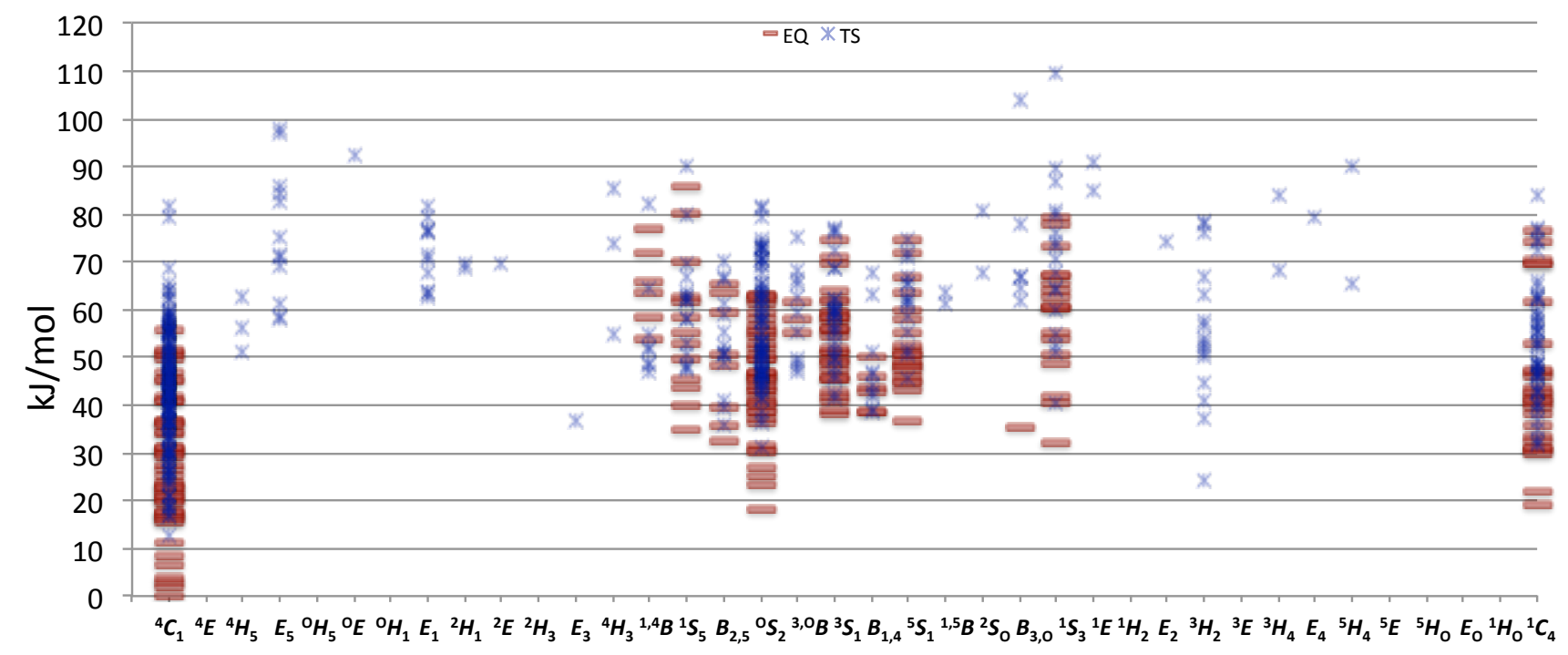

Figure S2-1. EQ structures (red bars) and TS structures (blue stars) classified into 38 conformational types are plotted against potential energy 